\title{
Traction forces: Stress fibres and the cortex work in tandem
}

\author{
Guillaume Charras ${ }^{1-3}$ \\ ${ }^{1}$ London Centre for Nanotechnology, University College London, 17-19 Gordon street, London, WC1H \\ $\mathrm{OAH}, \mathrm{UK}$ \\ ${ }^{2}$ Department of Cell and Developmental Biology, University College London, London, WC1E 6BT, UK \\ ${ }^{3}$ Institute for the Physics of Living Systems, University College London, London, WC1E 6BT, UK
}

\section{Stress fibres form a fully integrated meshwork with the submembranous contractile actin cortex that generates and propagates traction forces across the entire cell.}

Living cells generate surface forces through the action of myosin motor proteins on a biopolymer scaffold (known as filamentous actin or F-actin). Ever since the first observations of F-actin in cells, a challenge has been to understand how the organisation of the polymer at the molecular-scale controls force generation at the cellular-scale. Many of the cell's diverse actinous structures (lamellipodia, filopodia, stress fibres, etc) were first classified on the basis of morphology and, in an attempt to reduce complexity, these are often studied in isolation.

Stress fibres consist of linear arrays of actin filaments spanning most of the cell. They are bundled together by specialised crosslinking proteins, such as $\alpha$-actinin, and present a quasi-regular banding pattern of myosin along their length (Fig 1A), similar to the sarcomeres in muscle cells. Because of their suspected mechanical role, stress fibres have long been the topic of biophysical studies. Early studies employing 'wrinklable' substrates ${ }^{1}$ and, later, traction force microscopy ${ }^{2}$ suggested that stress fibres exert traction forces on the substrate. This was later confirmed by experiments in which cutting stress fibres with UV laser pulses decreased cellular traction stress ${ }^{3}$. Like sarcomeres, isolated stress fibres rapidly contract in the presence of ATP and divalent cations, applying forces on the order of 10 $\mathrm{nN}{ }^{4}$. More recent work using optogenetics to control myosin motor activity demonstrated that increasing contractility in one part of a stress fibre increases tension throughout the fibre ${ }^{5}$.

However, the relationship between stress fibres and other cellular F-actin networks has remained poorly understood. It is unclear if stress fibres are mechanically isolated from the rest of the cell, thus, only exerting stresses on the substrate between their extremities. Alternatively, stress fibres may be mechanically integrated with other cellular F-actin networks contributing to global surface tension. Such issues are key to our understanding of morphogenesis as well as cellular fate choice. Indeed, stem cell differentiation is sensitive to substrate stiffness ${ }^{6}$ that is sensed through traction forces. Some clues about this relationship have emerged from imaging the formation of stress fibres. These result from the merger of aligned filamentous structures ${ }^{7}$, known as radial fibres and transverse arcs, which arise from actin bundling induced by myosin-driven retrograde flow of F-actin from the cell periphery ${ }^{8}$. Thus, from genesis, stress fibres may be integrated with other cellular actin networks. Consistent with this, severing one stress fibre decreases traction stress in other stress fibres ${ }^{3}$, suggesting the existence of stress transmission mechanisms. However, the structure responsible for distributing stress is unknown.

In this issue of Nature Materials, Timothée Vignaud, Laetitia Kurzawa, and colleagues demonstrate that the submembranous actin cortex integrates stress fibres in a cell-wide meshwork that generates and transmits stresses across the cell Ref [A]. To demonstrate this, the authors use an ingenious combination of micropatterning, traction force microscopy, nanodissection, and computational modelling. Building on previous work, the authors plate cells on adhesive micropatterns designed to robustly position two prominent stress fibres along either side of each cell's long axis (Fig 1B). Surprisingly, severing one of these stress fibres dissipated significantly less than half of the total traction stress, contrary to expectations if stress fibres were the only structure generating traction. 
This suggested that the severed halves still remained under tension and, consistent with this, cutting the one of them released additional mechanical energy. Interestingly, after a cut, the amplitude of strain relaxation in the stress fibre decreased away from the location of the cut. As the stress fibre was not adherent to the substrate along its length, this indicated that it must be connected to the rest of the cell along its length and that these connections prevented relaxation.

Previous work proposed that discrete connections between stress fibres might serve to transmit stress. However, in the authors' experimental setup, the two stress fibres are far apart without any obvious connection. Alternatively, stress fibres might interact with the cortex, a thin isotropic network of actin bound to the cell membrane. As the cortex is less dense than stress fibres, its presence in fluorescence images of the actin cytoskeleton is easy to overlook. Yet, the cortex spans the whole cell and is a key contributor to surface tension. To test this idea, the researchers showed that stress fibres that had been isolated from the cortex by laser ablation released far more mechanical energy. As a proof of concept, the authors developed a minimal model of the cell consisting of two parallel stress fibres under tension connected by a network of springs. Comparison of experiments and model predictions revealed that tension generated by the cortex contributes significantly to the overall traction stresses exerted by the cell on its substrate. Transmission of stress occurs between the two actin structures because stress fibres are embedded in the actin cortex (Fig 2A).

The integration of stress fibres within the cortex poses a number of intriguing questions. It has long been known that stress fibre formation is induced by activation of $\mathrm{RhoA}^{9}$ but it is unclear if their assembly results from localised signalling coordinating the action of the proteins that make up stress fibres (Fig 2C). Alternatively, stress fibres may emerge from spontaneous alignment and aggregation of cortical actin filaments driven by a global increase in myosin activity (Fig 2B). Such contractilitydriven alignment has been observed during the formation of the pseudocleavage furrow in the $C$. elegans embryo ${ }^{10}$ and new work suggests that stochastic increases in contractility may drive the formation of stress fibres from cortical actin in spread cells ${ }^{11}$. More generally, as our understanding of the biophysics of the various cellular actinous structures grows, our next challenge will be to determine how they interact to dynamically control force generation and morphology in the whole cell.

The author declares no competing interests.

\section{References}

1 Harris, A. K., Stopak, D. \& Wild, P. Fibroblast traction as a mechanism for collagen morphogenesis. Nature 290, 249-251, doi:10.1038/290249a0 (1981).

2 Dembo, M. \& Wang, Y. L. Stresses at the cell-to-substrate interface during locomotion of fibroblasts. Biophys J 76, 2307-2316, doi:10.1016/S0006-3495(99)77386-8 (1999).

3 Kumar, S. et al. Viscoelastic retraction of single living stress fibers and its impact on cell shape, cytoskeletal organization, and extracellular matrix mechanics. Biophys J 90, 3762-3773, doi:10.1529/biophysj.105.071506 (2006).

4 Deguchi, S., Ohashi, T. \& Sato, M. Tensile properties of single stress fibers isolated from cultured vascular smooth muscle cells. J Biomech 39, 2603-2610, doi:10.1016/j.jbiomech.2005.08.026 (2006).

5 Oakes, P. W. et al. Optogenetic control of RhoA reveals zyxin-mediated elasticity of stress fibres. Nat Commun 8, 15817, doi:10.1038/ncomms15817 (2017).

6 Engler, A. J., Sen, S., Sweeney, H. L. \& Discher, D. E. Matrix elasticity directs stem cell lineage specification. Cell 126, 677-689, doi:10.1016/j.cell.2006.06.044 (2006).

$7 \quad$ Hotulainen, P. \& Lappalainen, P. Stress fibers are generated by two distinct actin assembly mechanisms in motile cells. J Cell Biol 173, 383-394, doi:10.1083/jcb.200511093 (2006). 
8 Verkhovsky, A. B., Svitkina, T. M. \& Borisy, G. G. Myosin II filament assemblies in the active lamella of fibroblasts: their morphogenesis and role in the formation of actin filament bundles. J Cell Biol 131, 989-1002, doi:10.1083/jcb.131.4.989 (1995).

9 Chrzanowska-Wodnicka, M. \& Burridge, K. Rho-stimulated contractility drives the formation of stress fibers and focal adhesions. J Cell Biol 133, 1403-1415, doi:10.1083/jcb.133.6.1403 (1996).

10 Reymann, A. C., Staniscia, F., Erzberger, A., Salbreux, G. \& Grill, S. W. Cortical flow aligns actin filaments to form a furrow. Elife 5, doi:10.7554/eLife.17807 (2016).

11 Lehtimaki, J. I., Rajakyla, E. K., Tojkander, S. \& Lappalainen, P. Generation of stress fibers through myosin-driven re-organization of the actin cortex. BioRxiv, doi:https://doi.org/10.1101/2020.06.30.179283 (2020).

12 Peterson, L. J. et al. Simultaneous stretching and contraction of stress fibers in vivo. Mol Biol Cell 15, 3497-3508, doi:10.1091/mbc.e03-09-0696 (2004). 
Figures:

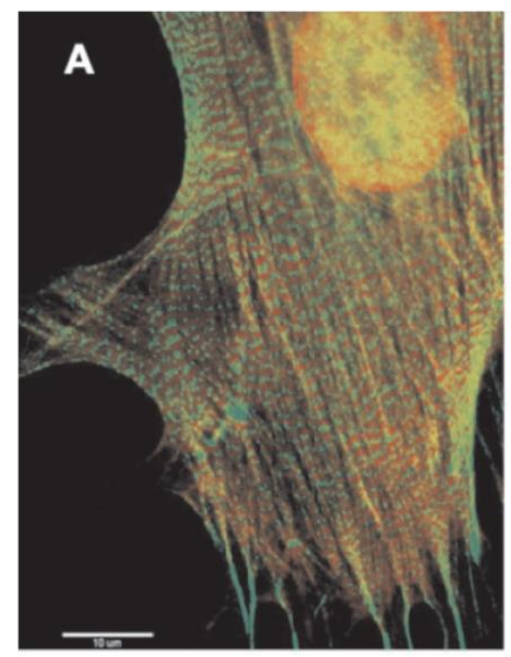

B
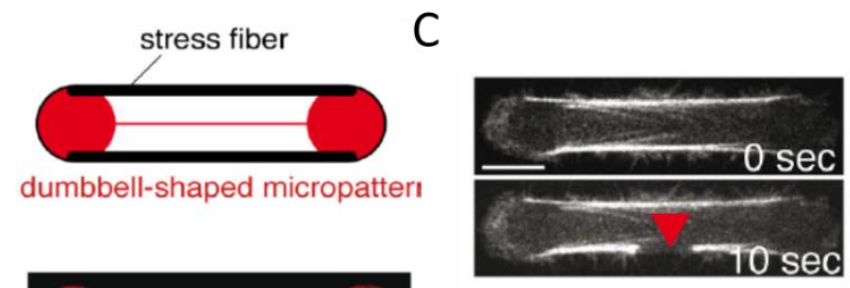
micropattern

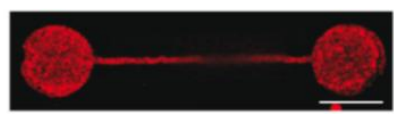

F-actin
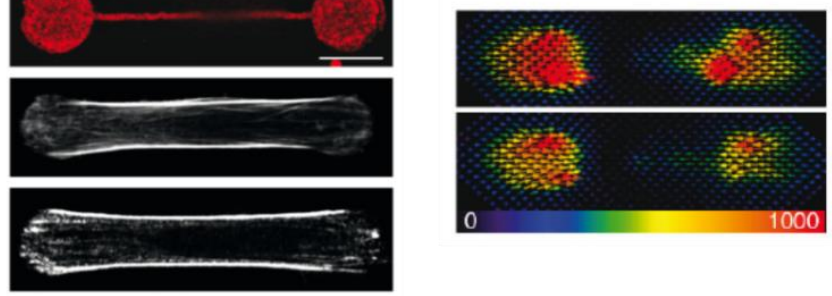

phosphorylated myosin light chain

D

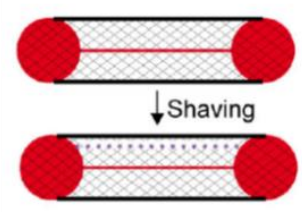

Fiber shaving
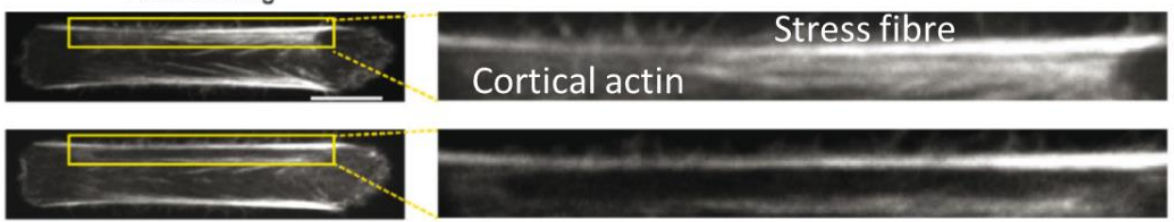

Figure 1: Stress fibres collaborate with the submembranous actin cortex to generate traction forces. A. Stress fibres present an alternating arrangement of myosin (red) and $\alpha$-actinin (green) along their length in Swiss 3T3 cells plated on glass substrates. Image obtained from ${ }^{12}$. B. Plating cells on dumbbell-shaped micropatterns generates two prominent stress fibres on each of the long cell edges. C. Laser ablation of a stress fibre releases mechanical energy but the cut stress fibre retains some tension. Top: F-actin localisation before (0s) and after (10s) laser ablation. The red arrow indicates the location of the cut. Bottom: Traction stress field before and after ablation. D. Disconnecting the stress fibre from the cortex along its whole length using a pulsed UV laser ("shaving") releases mechanical energy. Following shaving, more mechanical energy is released when the fibre is cut as in C. (A-D) Scale bars $=10 \mu \mathrm{m}$. (B-D adapted from Ref[A] with permission). 


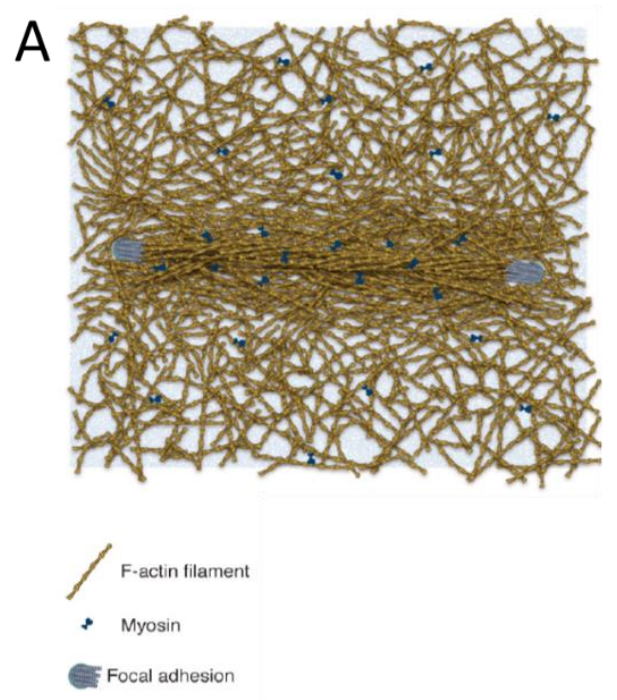

B

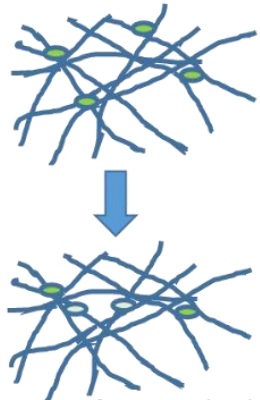

Turnover of myosins leads to a local increase in myosin density

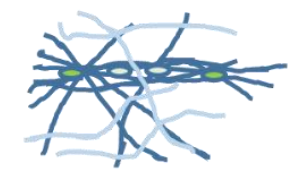

Myosin motor activity aligns filaments to create a stress fibre

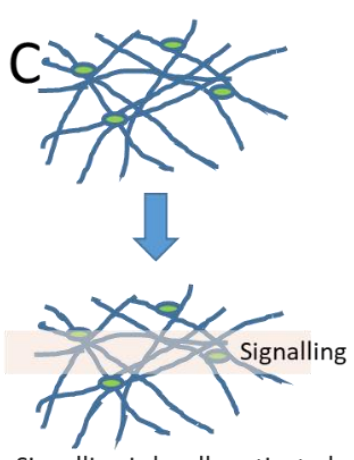

Signalling is locally activated
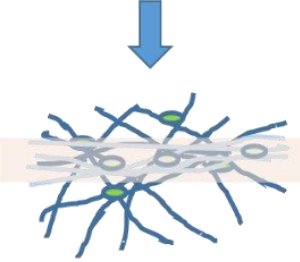

In the high signalling activity region: New filaments are nucleated New motors are recruited A stress fibre is formed

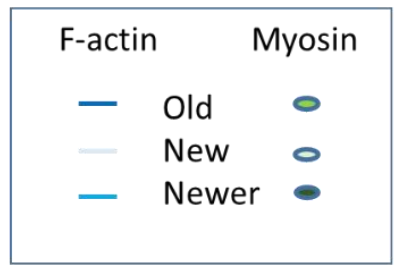

D

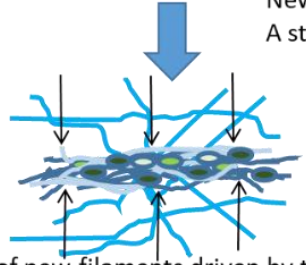

Flow of new filaments driven by the locally higher myosin contractility increases the density of F-actin and myosin in the fibre. Banding appears.

Figure 2: Stress fibres are mechanically integrated within the submembranous actin cortex. A. Schematic of the integration of a stress fibre within the submembranous cortex. (B-D) Hypothetical mechanisms of stress fibre formation in the actin cortex. B. Stochastic formation model. Because of turnover of myosin minifilaments, a locally higher density of myosin mini-filaments stochastically arises. Contractility of the myosins leads to alignment of the actin filaments creating a nascent stress fibre. C. Localised signalling model. A localised increase in signalling at the membrane leads to locally higher nucleation of new actin filaments and recruitment of myosins. D. The locally higher tension created in the nascent stress fibre drives flow of additional filaments and myosins (black arrows), leading to growth of the fibre by accretion from the surrounding cortex. 